\title{
Flujos Doppler e imágenes que se deben conocer. Parte 1: flujo Doppler arterial de vasos del cuello y extremidades
}

Adrián H. D'Ovidio*
Gabriel Perea**
Patricio Glenny***
Laura Titievsky

* Cardiología e Imagen Cardiovascular. MTFAC, MGTR, FACC, FSISIAC. Hospital Rawson. San Juan. Argentina

** Cardiología e Imagen Cardiovascular. Instituto Cardiovascular de Buenos Aires. Argentina

*** Cardiología e Imagen Cardiovascular. Jefe de Imágenes Cardiovasculares, Instituto Cardiovascular Adventista. Argentina

**** Cardiología e Imagen Cardiovascular. Instituto de Cardiología de Corrientes. Argentina

Recibido: 29/09/2020

Aceptado: 13/10/2020

En línea: 31/12/2020

Citar como: D'Ovidio AH, Perea G, Glenny P, Titievsky L. Flujos Doppler e imágenes que se deben conocer. Parte 1: flujo Doppler arterial de vasos del cuello y extremidades. Rev Ecocar Pract (RETIC). 2020 (Dic); 3 (3): 36-42. doi: 10.37615/retic.v3n3a2.

Cite this as: D'Ovidio AH, Perea G, Glenny P, Titievsky L. Doppler flows and images to know. Part 1: arterial Doppler flow of vessels of the neck and extremitie. Rev Ecocar Pract (RETIC). 2020 (Dic); 3 (3): 36-42. doi: 10.37615/retic.v3n3a2.

\section{Palabras clave}

$\triangleright$ Ultrasonido vascular

$\triangleright$ Doppler carotídeo

$\triangleright$ Doppler de miembros

\section{RESUMEN}

La ecografía Doppler vascular, en todas sus formas, permite evaluar todos los territorios arteriales y venosos del organismo, con las ventajas de su elevadísima sensibilidad y especificidad, y valores predictivos positivo y negativo, bajo costo, reproducibilidad, total disponibilidad y portabilidad de los equipos, lo que permite efectuar estudios en cabecera del paciente. Como todo método que emplee ultrasonido, es operador dependiente, y por eso, lograr alcanzar la acreditación para poder hacer correctamente estos estudios lleva mucho tiempo de estudio y capacitación práctica. Ser un "observador de flujos" es clave para un buen ecografista vascular, ya que los flujos "hablan", nos dan información valiosísima que permite un gran acercamiento diagnóstico. En esta primera entrega de los "Flujos Doppler e imágenes que se deben conocer", se abordarán el estudio de arterias del cuello y de arterias de miembros. En próximas publicaciones, se desarrollarán el resto de territorios arteriales y venosos.

ABSTRACT
The vascular Doppler ultrasound in all its forms allows to evaluate all the arterial and venous territories of the or-
ganism, with the advantages of its very high sensitivity and specificity and positive and negative predictive values,
its low cost, reproducibility, full availability, equipment portability, allowing for "bed side" studies. Like any method
that uses ultrasound, is an operator dependent and therefore achieving accreditations in order to do these studies
correctly takes a long time of study and practical training. Being a "flow observer" is key to a good vascular sono-
grapher because the flows "speak", give us valuable information that allows a great diagnostic approach. This is
the first delivery of the "Doppler flows and images to be known", the study of arteries of the neck and arteries of
extremities will be addressed. Upcoming publications will include the rest of the artery and venous territories.

\section{Introducción}

El ultrasonido vascular es una herramienta de muy alta sensibilidad y especificidad diagnóstica, con la posibilidad de encontrar el sitio específico de la/las obstrucción/obstrucciones y determinar claramente su localización, extensión, morfología de placa, severidad, compromiso distal o proximal y presencia o no de colaterales.

Es generalmente, en todas sus formas (modo B, Doppler pulsado, Doppler color, power Doppler, B-flow, angio-B flow, eco 3D), el primer método empleado para evaluar pacientes con enfermedad arterial periférica (EAP).
La sangre fluye por la energía creada por la contracción cardíaca, haciéndola llegar a todo el organismo. El flujo depende básicamente de dos factores determinantes: la energía disponible para impulsar el flujo sanguíneo, y la resistencia al flujo que presenta el sistema vascular. Es un sistema complejo de juego de presiones y energías en movimiento constante en un sistema cerrado que funciona siguiendo las leyes de la hidrodinámica ${ }^{(1,2)}$.

\section{Fisiología del flujo vascular}

El flujo arterial es pulsátil y es el resultado del volumen sistólico, que a su vez está determinado por cuatro componentes: 
- Precarga (o tensión de fin de diástole ventricular). Sus componentes son e retorno venoso (que depende básicamente de la viscosidad; de las presiones intrapleural, abdominal y pericárdica; y de la bomba muscular venosa), la contracción atrial y la distensibilidad ventricular.

- Poscarga (o tensión durante el periodo eyectivo). Tiene un componente externo, constituido por la resistencia periférica determinada por las arteriolas y la impedancia aórtica, y un componente interno, que no es otro que la tensión sobre la pared o estrés sistólico.

- Contractilidad o estado inotrópico.

- Frecuencia cardíaca.

El volumen sistólico multiplicado por la frecuencia cardíaca da como resultado el gasto cardíaco; y dividido por la superficie corporal da como resultado el índice cardíaco. Por último, la combinación del volumen minuto y la resistencia periférica determinan la tensión arterial|(3)

La actividad pulsátil cardíaca requiere un sistema arterial que amortigüe las pulsaciones y transforme un flujo de alta presión y pulsátil en un flujo continuo de baja presión. Para ello existen tres elementos fundamentales(3):

- Efecto amortiguador y de receptáculo de los grandes vasos. Con su gran distensibilidad, ecualizan la variación cíclica de la presión y flujo.

- Sistema disipador de energía. Constituye la red arteriolar periférica, que completa la reducción de la presión hasta el nivel capilar.

- Sistema de capacitancia venosa. Mantiene el llenado continuo y evita la sobrecarga de volumen.

Comprender el acoplamiento ventrículo-arterial llevó centenares de años y pruebas. El aparato circulatorio consiste en una bomba encargada de mantener la circulación sanguínea a través de un sistema de distribución y circulación que permite la adecuada oxigenación y depuración sanguíneas acorde con la demanda tisular periférica(3)

Como se puede observar, son múltiples las variables que determinan los flujos arterial y venoso, a las que debemos sumar el endotelio y el músculo liso.

Cuando se evalúa un flujo arterial con Doppler pulsado, el flujo es el resultado de todo lo que ocurre antes del lugar en el que tenemos colocado el volumen de muestra a nivel de dicha ubicación siguiendo la ley de conservación de la masa, la energía y el momento de Lavoisier ${ }^{(1-5)}$.

Resulta fundamental conocer, además, que para que la sangre circule tiene que existir una diferencia de presión, en el caso de la sangre de presión media y que siguiendo la ley de Hagen-Poiseuille permite la circulación en un sentido. Se relaciona con las paredes distensibles de los vasos sanguíneos de acuerdo con la ley de Laplace (Tensión = Presión x Radio del vaso/2 espesor parietal).

Siguiendo el principio de Bernoulli y su teorema, que dice que "en un sistema ideal sin rozamiento ni viscosidad, la energía que posee un fluido permanece constante en todo su recorrido", y que la presión hemodinámica puede ser definida a través de la suma de la presión hidrostática $(P)$ y la presión cinética (densidad por velocidad) $(P+1 / 2 \delta V 2=$ constante), y que resulta fundamental para comprender el comportamiento del flujo y la presión en las diferentes secciones vasculares y zonas de estenosis y postestenosis; cuanto menor sea la velocidad, mayor es la presión hidrostática, la velocidad será mayor cuanto menor la sección del vaso que se estudie. Pero en el segmento posterior a una obstrucción la presión hidrostática es menor, debido a que en la obstrucción hubo una pérdida o disipación de energía.

En eco Doppler vascular utilizamos transductores lineales de alta frecuencia entre 4-12 MHz. Para ecografía esplácnica y de la aorta abdominal e ilíacas utilizamos un transductor Convex de 3-5 MHz, y los transductores sectoriales que los cardiólogos utilizamos habitualmente (2 a 4-5 MHz) también son clave al evaluar algunos territorios, como los troncos supraaórticos y, particularmente, la arteria subclavia izquierda.

Desde el punto de vista técnico, es fundamental utilizar un ángulo adecuado de incidencia, estamos incidiendo el vaso perpendicularmente y la velocidad registrada tanto con Doppler pulsado como con Doppler color por tanto sería $0^{\circ}$. Así, se utiliza en todo el mundo un ángulo de incidencia de $60^{\circ}$, estudiamos de este modo velocidades radiales y no reales (éstas son mayores, pero con diferencias no significativas).

Con eco Doppler, evaluando los flujos tanto con Doppler pulsado como continuo (ciego en vascular) podemos determinar el flujo a través de la fórmula: Flujo $=$ VTI $x$ Área, donde VTI es la integral de velocidades en el tiempo, y el área se obtiene a través de la fórmula $\pi . r 2$.

Habitualmente, utilizamos volúmenes de muestra pequeños (1-1.5 mm), que se colocan en el centro del vaso para tomar las velocidades más altas tanto de los flujos laminares como de los turbulentos, evitando así tomar todas las velocidades, especialmente las más cercanas a las paredes de los vasos que son más lentas.

Es importante distinguir entre flujos de alta y de baja resistencia (Figura 1):

- Flujos de alta resistencia (aorta, arterias ilíacas interna y externa, arterias de miembros superiores e inferiores, arteria carótida externa). Se caracterizan por tener una rampa ascendente empinada, un rápido descenso, que pasa la línea de base en la protodiástole y se hace nuevamente positiva e incluso nuevamente negativa en la telediástole. Esto es por el llamado "efecto Windkessel" (atizador de fuego). La aorta, la arteria con mayor distensibilidad del organismo, en la protosístole, además de dejar pasar el 70\% del flujo, se distiende, esa sangre "retenida" en la aorta proximal, a nivel de su segmento sinusal, genera energía potencial, al cerrarse la válvula aórtica porque la resistencia periférica sumada a la impedancia aórtica superan a la presión ventricular izquierda de fin de diástole, se transforma en energía cinética, llenándose las arterias coronarias y manteniendo el flujo arterial en cada segmento durante toda la diástole ventricular.

- Flujos de baja resistencia. Tienen una rampa de ascenso sistólica menos empinada, en general tienen velocidades levemente menores, y el flujo continúa durante toda la diástole hasta el inicio de la siguiente sístole, es decir, tienen flujo diastólico registrable y que no alcanza la línea de base. Es el flujo típico de las arterias carótida interna, vertebral, las arterias cerebrales, las renales... Para estudiar de manera práctica en personas normales la diferencia entre ambos patrones es muy útil utilizar el estudio de vasodilatación mediada por flujo.

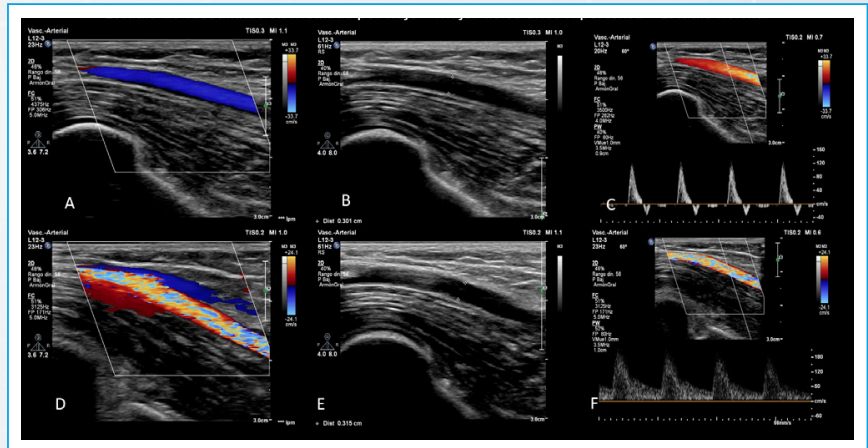

Figura 1. Cambio del tipo de flujo de alta a baja resistencia por la vasodilatación mediada por flujo

En las arterias, cada territorio tiene una onda propia diferente definida por una propiedad muy importante del flujo conocida como pulsatilidad (Figura 2). Las ondas Doppler pueden tener pulsatilidad baja, mediana o alta: 
- Ondas Doppler de baja pulsatilidad. Tienen picos sistólicos amplios y flujo anterógrado durante todo el ciclo cardíaco, desde la sístole hasta la telediástole; con ondas monofásicas porque estos vasos alimentan sistemas circulatorios de baja resistencia al flujo, son típicas de las arterias carótida interna, vertebral, renales y de todas las arterias cerebrales.

- Ondas Doppler de moderada pulsatilidad. Tienen una morfología que está a mitad de camino entre los flujos típicos de baja resistencia y los de alta resistencia, como la resistencia al flujo es moderada el pico sistólico se haya alto y afilado, pero existe flujo anterógrado durante toda la diástole, que puede ser interrumpido por una inversión del flujo al inicio de la misma. Son flujos típicos de la arteria carótida externa y la mesentérica superior.

Ondas Doppler de alta pulsatilidad. Tienen picos altos, estrechos y con rampa ascendente y descendente recta (afilados) durante la fase sistólica con flujo diastólico invertido, que puede tener dos fases o tres (véase más adelante al describir el flujo normal arterial periférico). Es un flujo típico de las extremidades superiores e inferiores en reposo.

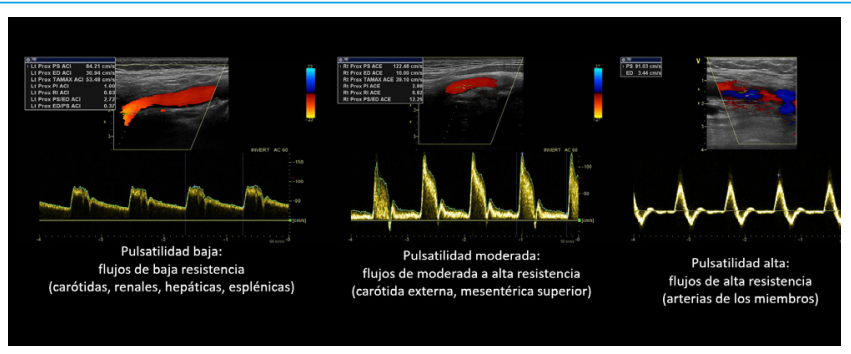

Figura 2. Pulsatilidad de flujos arteriales

Las mediciones más útiles para determinarlos son el índice de pulsatilidad (IP) de Gosling (Velocidad pico sistólica [VPS] - Velocidad pico diastólica [VPD] / Velocidad media), el índice de resistencia de Pourcerot (VPS - VPD / VPS) y la relación sistólica/diastólica. El IP es probablemente el índice más empleado, se puede utilizar para cuantificar el grado de amortiguación de la onda de pulso en diferentes lugares. El flujo amortiguado distal a una obstrucción severa tendrá un IP más bajo que una onda normal.

Las condiciones de pulsatilidad pueden alterarse en situaciones tanto fisiológicas como patológicas. Fisiológicamente, con el calor intenso, el ejercicio enérgico o procesos infecciosos distales, todos procesos que generan vasodilatación distal significativa y que pueden hacer que el flujo tri/tetrafásico pase a ser durante un tiempo breve monofásico. En condiciones patológicas, el flujo distal a una obstrucción significativa en reposo es monofásico (secundario a obstrucción proximal) y característicamente se haya "amortiguado", con ascenso lento (rampa ascendente lenta) y tiene flujo anterógrado durante toda la diástole. Más adelante se describe en detalle el flujo típico postobstrucción significativa y se muestran ejemplos de condiciones fisiológicas y patológicas.

\section{Eco Doppler de las arterias del cuello}

Si observamos un estudio de eco Doppler de vasos del cuello normal (Figura 3), veremos que las arterias carótida interna y vertebral tienen flujos típicos de baja resistencia, ya que se dirigen sin dar ramificaciones en el cuello directamente a la cavidad craneana para dar origen a las arterias basilar (arteria vertebral) y cerebrales media, anterior y oftálmica (carótida interna) irrigando un territorio como el cerebral que requiere baja pulsatilidad, flujo durante todo el ciclo cardíaco, y que ofrece muy baja resistencia y presenta gran colateralidad. La arteria carótida externa tiene un flujo típico de alta resistencia (da todas sus ramas en el cuello para irrigar cara, glándulas, músculos, lengua, etc.), y la arteria carótida común tiene un flujo "intermedio" entre resistencias bajas $y$ altas $^{(51-8)}$.

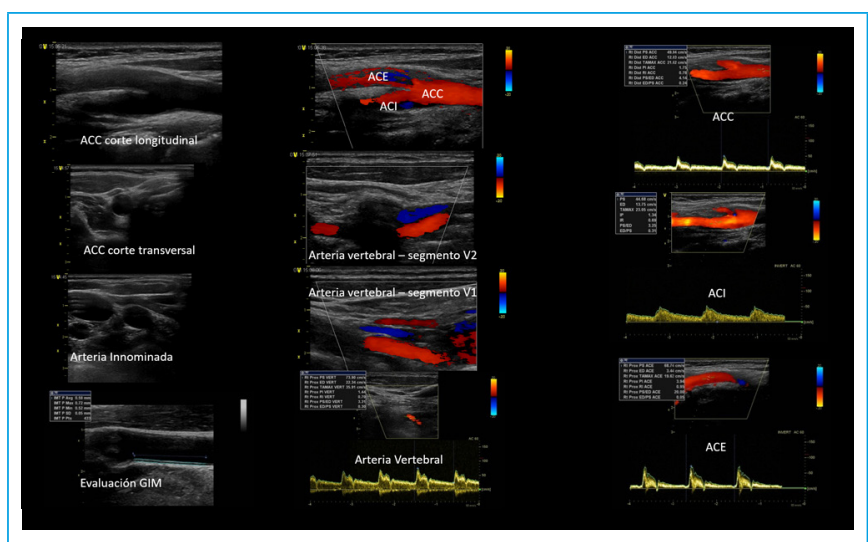

Figura 3. Estudio normal de arterias del cuello. Eco bidimensional, Doppler color y Doppler pulsado (ACC: arteria carótida común; ACE: arteria carótida externa; ACl: arteria carótida interna; GIM: grosor íntimo-medial)

Una de las formas de evaluar la significación de las placas arterioescleróticas en las arterias (además de la caracterización de las placas) es la valoración de las velocidades pico sistólicas (VPS) y diastólicas (VPD). A medida que el diámetro de una arteria se va reduciendo, para mantener el flujo constante, las VPS y VPD se van incrementando paulatinamente, superando la velocidad de $125 \mathrm{~cm} / \mathrm{s}$ (en condiciones basales) pero sin alcanzar los $230 \mathrm{~cm} / \mathrm{s}$. Recién cuando la reducción del diámetro del vaso alcanza aproximadamente el 50\% (lo que corresponde a una reducción del área de aproximadamente 70\%), el flujo comienza a caer a pesar del aumento de la velocidad; éste es el "punto de significación". Hasta este punto, las velocidades aumentadas sugieren que las obstrucciones son leves (menores del 49\%) (Figura 4).

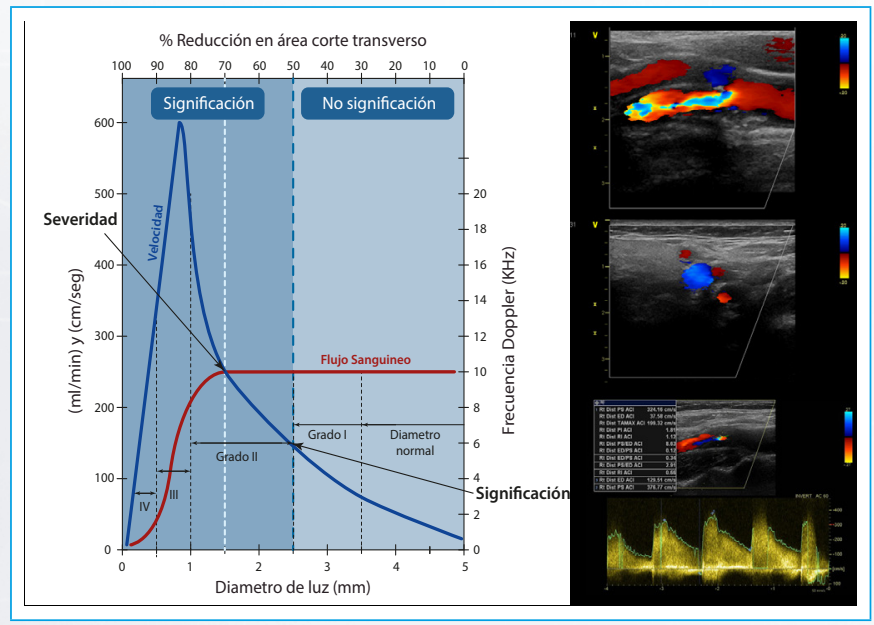

Figura 4. Relaciones entre la velocidad del flujo, flujo sanguíneo, área y diámetro arterial. El"punto de significación"separa al gráfico en dos sectores: el de la derecha, de "no significación", donde las placas no reducen el flujo sanguíneo a los tejidos y se compensa con un aumento no significativo de las velocidades; y otro, "de significación", donde a pesar del aumento de las velocidades el flujo comienza a caer. Las VPS mayores de $125 \mathrm{~cm}$ y menores de $230 \mathrm{~cm} / \mathrm{s}$ determinarán la presencia de "placa significativa; de grado moderado (reducción del diámetro entre 50-69\%)", aquellas que superen los $230 \mathrm{~cm} / \mathrm{s}$ de VPS y $100 \mathrm{~cm} / \mathrm{s}$ de VPD se considerarán placas significativas, de grado severo y tendrán un cociente (VPS obstructiva/VPS preobstructiva $>4)^{(1,2,9)}$.

Al superar el punto de significación y alcanzar la reducción de más del 70\% del diámetro (lo que supone reducción del 90\% del área), se observa que las velocidades se elevan significativamente y, a pesar de ello, la caída del flujo sanguíneo es marcada; éste es el "punto de severidad" y se alcanza cuando las velocidades (en condiciones basales) superan los $230 \mathrm{~cm} / \mathrm{s}$ de VPS y $100 \mathrm{~cm} / \mathrm{s}$ de VPD. Muy importante además es calcular la relación de flujo postobstruc- 
ción con el flujo previo, que será significativo cuando supere 3.5 y severo al alcanzar el valor de 4

Las guías actuales ${ }^{(10-16)}$ sugieren ser más específicos con las placas significativas de grado severo. Si la VPS alcanza $240 \mathrm{~cm} / \mathrm{s}$ y la VPD no supera los 100 $\mathrm{cm} / \mathrm{s}$ y el cociente de VPS intra y preobstrucción es $\geq 2$ pero sin alcanzar 4 la obstrucción será del 60\%; si la VPS supera $230 \mathrm{~cm} / \mathrm{s}$ y hasta los $370 \mathrm{~cm} / \mathrm{s}$, la VPD es $>100 \mathrm{~cm} / \mathrm{s}$ y el cociente $>4$ y la VPS postobstrucción es $\geq 50 \mathrm{~cm} / \mathrm{s}$, la placa obstruirá el vaso en un $70 \%$; al alcanzar $370 \mathrm{~cm} / \mathrm{s}$ y observar colateralidad de ser posible, el cociente $>4$ y la velocidad postobstrucción $<50 \mathrm{~cm} / \mathrm{s}$ será del 80\%; luego ya hablamos de suboclusiones (obstrucciones 90-99\%) con VPS postobstrucción $<30 \mathrm{~cm} / \mathrm{s}$; y luego de oclusión (Figura 5).

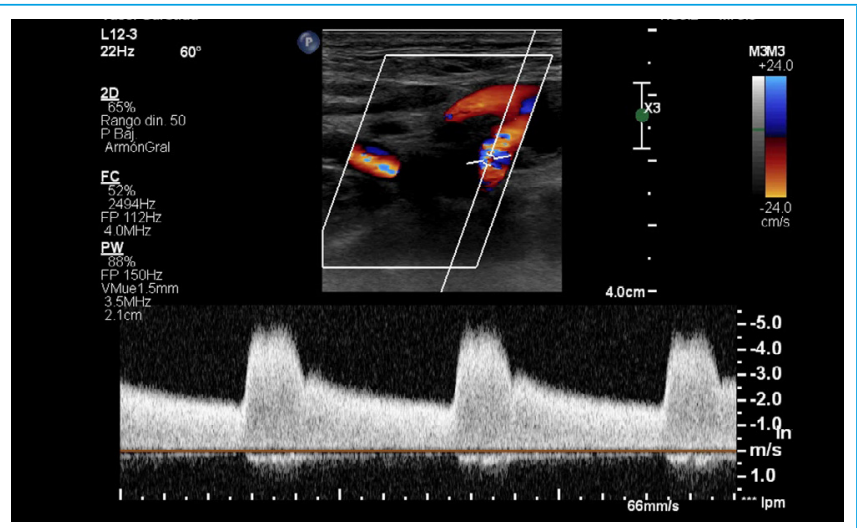

Figura 5. Eco Doppler típico de una placa significativa, severa, tipo III-IV en el origen de la arteria carótida interna

En la Figura 6, Figura 7 y Figura 8 se muestra un flujo típico que se observa en la arteria vertebral en pacientes con "robo subclavio". Se denomina "robo subclavio", o "robo de la subclavia" a aquella situación clínica en la que, como consecuencia de una estenosis significativa de la arteria subclavia antes de dar la rama vertebral, se produce flujo reverso en la arteria vertebral ipsilateral. Dicho flujo reverso se origina en que desde la arteria vertebral opuesta y a través del tronco basilar, la vertebral ipsilateral a la obstrucción presenta flujo reverso parcial o total, de acuerdo con el grado de obstrucción ${ }^{(17,18)}$. El robo subclavio izquierdo se observa en el 90\% de los casos y el derecho sólo en el 10\%. Los robos subclavios no son un hallazgo frecuente, pero deben tenerse en cuenta. Se han descrito en la literatura diferentes tipos; tipos de Vollmar:

- Vertebro-vertebral.

- Carotídeo-basilar.

- Carótida externa-vertebral.

- Carotídeo-subclavio (sólo ocurre con oclusión del TBC).

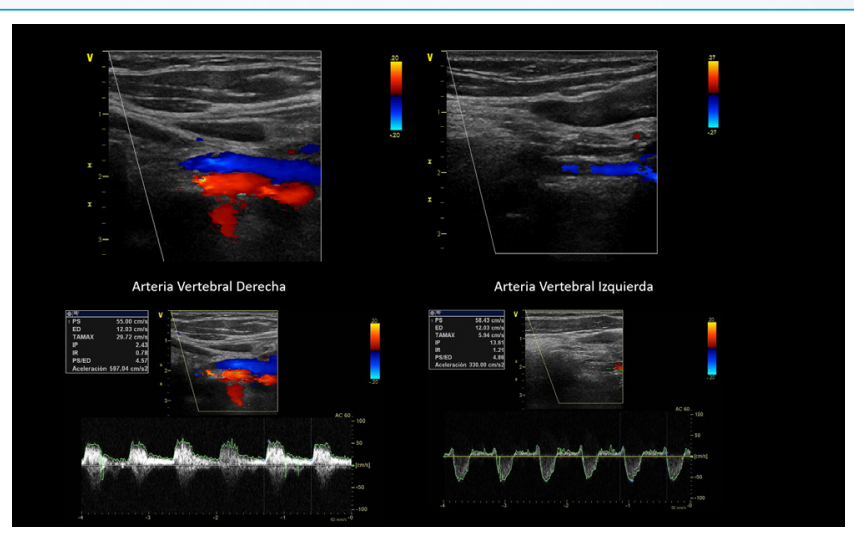

Figura 6. Robo subclavio izquierdo

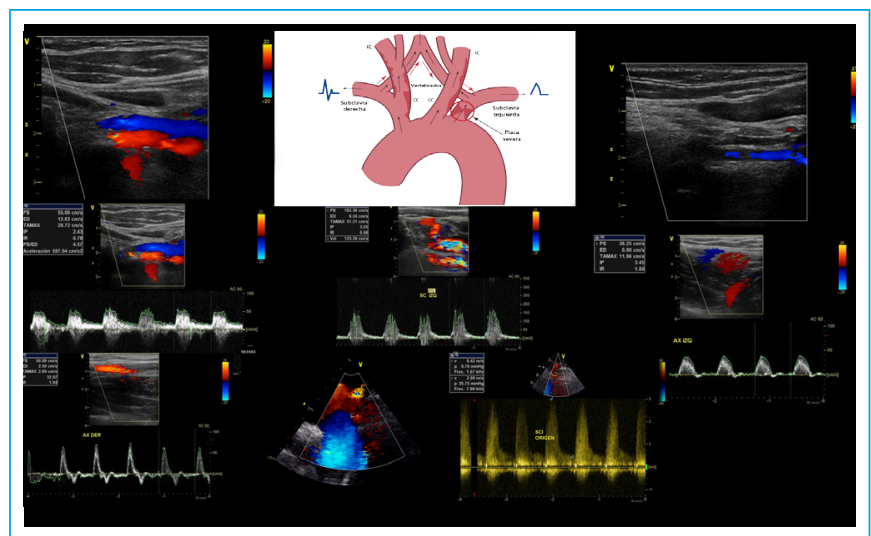

Figura 7. Robo subclavio izquierdo. Placa severa en el origen de la arteria subclavia izquierda y flujo típico de robo tipo 2 en la arteria vertebral izquierda

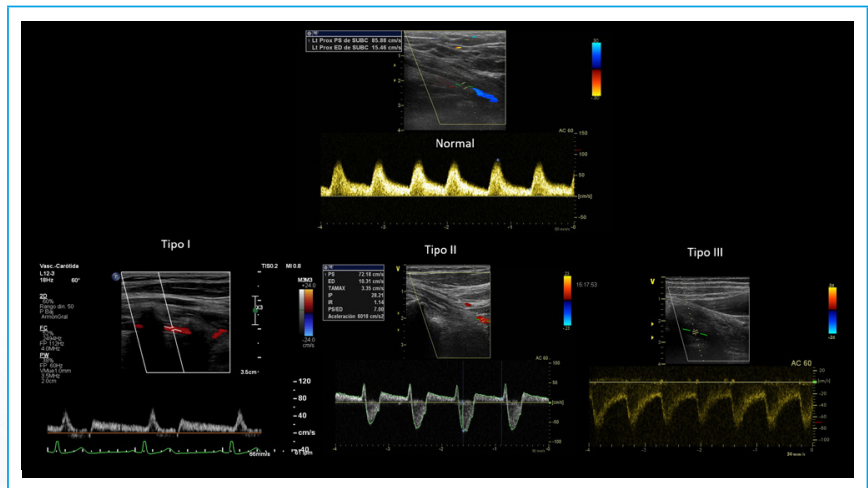

Figura 8. Robo Tipos de robo subclavio

Los cambios que se hallan en la evaluación de los vasos del cuello con eco Doppler generan un patrón característico, que debe conocerse, y su detección orienta directamente al diagnóstico. La evaluación completa de los flujos de los vasos del cuello, troncos supraaórticos y arterial de los miembros superiores permite no sólo hacer el diagnóstico del robo subclavio, sino hallar la ubicación precisa de la obstrucción y evaluar sus consecuencias hemodinámicas(19). De acuerdo con ellas, se reconocen tres grados de robo subclavio (hemodinamia de la arteria vertebral [Branchereau]):

- Estadio I. Flujo anterógrado vertebral reducido.

- Estadio II. Flujo reverso con el estudio de hiperemia reactiva.

- Estadio III. Flujo vertebral retrógrado permanente.

Además, se han descrito tres tipos de acuerdo con la severidad de la obstrucción de la arteria subclavia ipsilateral y, por tanto, del grado de flujo retrógrado ${ }^{(18)}$ :

- Tipo I. Mínimos cambios hemodinámicos. El eco Doppler muestra flujo anterógrado con leve desaceleración medio diastólica, que puede tornarse flujo retrógrado en respuesta al test de hiperemia reactiva en el brazo ipsilateral a la oclusión.

- Tipo II. Cambios hemodinámicos moderados. El eco Doppler muestra flujo reverso parcial.

- Tipo III. Se presenta flujo retrógrado de manera permanente.

\section{Eco Doppler de las arterias de las extremidades}

El flujo normal en las arterias periféricas es característicamente tri/tetrafásico, de alta resistencia, con una onda sistólica predominante seguida de dos o tres ondas diastólicas que se expresarán en las arterias normales y que expresan las 
propiedades de distensibilidad de las arterias (efecto Windkessel). Las velocidades normales siempre son menores de aproximadamente $1,5 \mathrm{~m} / \mathrm{s}$ y a medida que las arterias se van estrechando por la presencia de placas suceden cambios muy importantes en el flujo. Cuando la luz se ve reducida en su diámetro en un 50\% (corresponde a una reducción del área de 70\%), el flujo aumenta sus velocidades proporcionales al grado de obstrucción, va a aumentar la velocidad diastólica hasta tener un flujo monofásico de velocidad muy alta en la zona de la estrechez muy severa. Como dato importante, el eco Doppler tiene para obstrucciones significativas una sensibilidad y especificidad superiores al 90\%. La presencia en las lesiones muy severas o críticas de "imagen especular" en el flujo sistólico es otro dato predictivo de severidad, pero el más importante sin duda porque es útil hasta en las situaciones de bajo gasto cardíaco o lesiones "en tándem" es que la relación entre las VPS a nivel de la obstrucción y previo a ella sea $\geq 4$, lo que sugiere severidad de la obstrucción ${ }^{(13-18,20-27)}$ (evidencia IB, guías europeas) (Figura 9 a Figura 11).

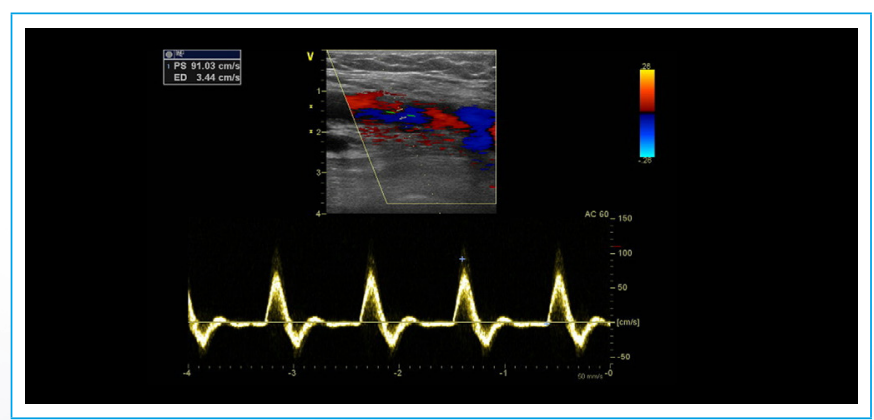

Figura 9. Flujo trifásico normal de arterias periféricas (típico de arterias de miembros inferiores y superiores)

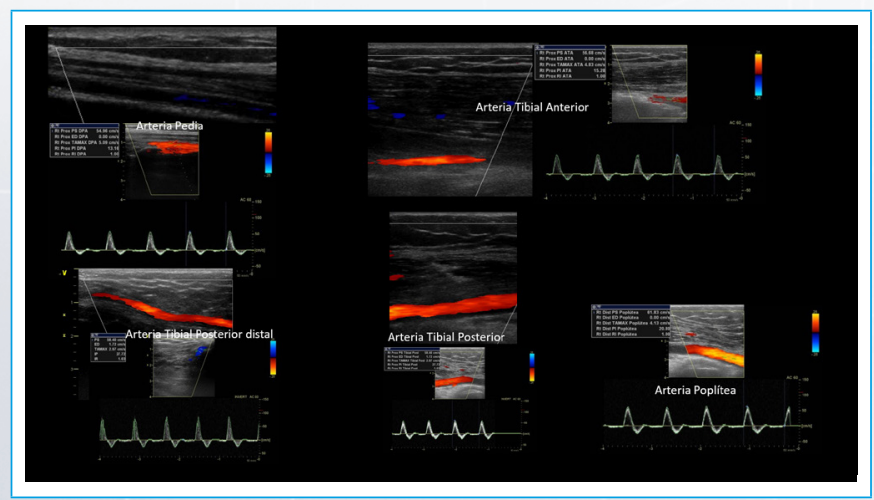

Figura 10. Flujos normales en todas las arterias del miembro inferior

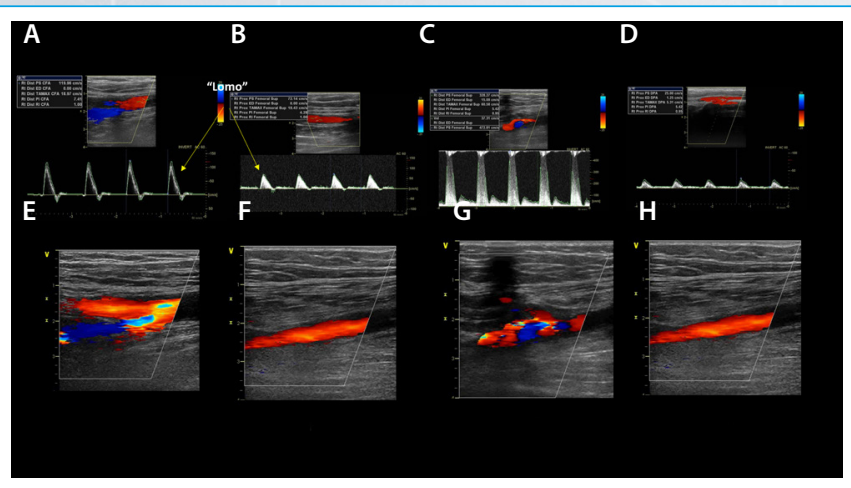

Figura 11. Arteriopatía obstructiva severa crónica. Obsérvese el flujo turbulento con obstrucción severa a nivel del tercio distal de la femoral superficial. Los cambios preobstrucción (reducción de la diástole y aparición del "lomo" en la rampa descendente del flujo [A, B, E, F]) y los cambios postobstrucción con flujo monofásico "amortiguado" por obstrucción superior (C, D, G, H)
Antes de la estrechez severa, el flujo va perdiendo progresivamente sus componentes diastólicos, aparece en la rampa descendente del flujo sistólico un "lomo" característico, en lugar del rápido y "recto" descenso normal, sugiriendo que existe una obstrucción significativa más distal cercana.

Distal a la obstrucción severa, se evidencia reducción muy significativa del flujo (y de la presión distal a la obstrucción que se expresará por un índice tobillobrazo $<0,9)$ que será monofásico, de baja velocidad, secundario a obstrucción proximal.

Además, en el mismo momento el operador entrenado en todos los territorios vasculares podrá chequear con el transductor convexo la aorta abdominal y arterias ilíacas, buscando placas y aneurismas (evidencia lla-B, de las guías americanas) asintomáticos de la aorta abdominal, presentes en el 10\% de los pacientes con EAP significativa, y con el mismo transductor lineal evaluar las arterias del cuello (carótidas y vertebrales), teniendo así un screening vascular completo $^{(16-19)}$

Si se observan las Figuras 12 a 14, se verá el caso de una paciente que ingresa al laboratorio, no se halla patología arterial periférica tanto clínica como con eco 2D y Doppler color, pero el flujo en las arterias pedias es monofásico. Pasan unos minutos y el flujo en ambas arterias pasa a ser trifásico. Se trataba de vasodilatación, como se explicó más arriba, obsérvese el ascenso y descenso rápidos, las velocidades normales, la "ventana acústica" que indica flujo laminar, para diferenciarlo como se observa en la Figura 14 del flujo monofásico por obstrucción superior, que tiene baja velocidad, no tiene ventana acústica, tiene rampa ascendente lenta y se halla placa severa previa.

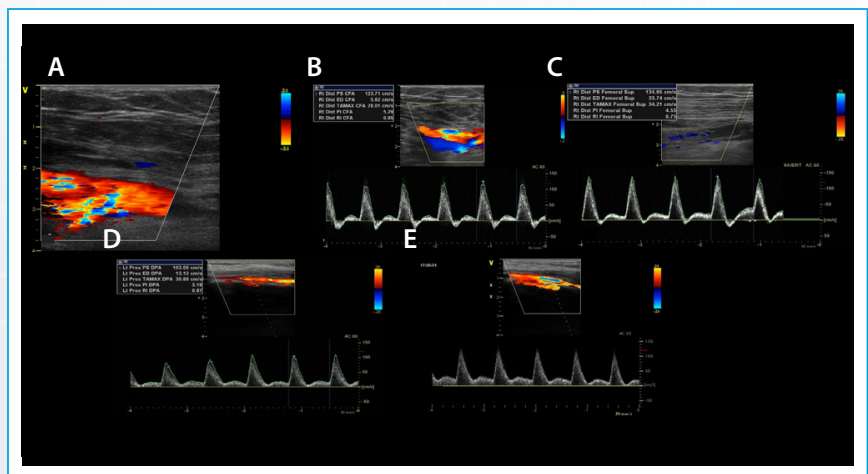

Figura 12. Estudio de eco Doppler arterial normal de miembros: flujo trifásico en arteria femoral común (A, B), y femoral superficial (C); flujo monofásico en las arterias pedias (D, E)

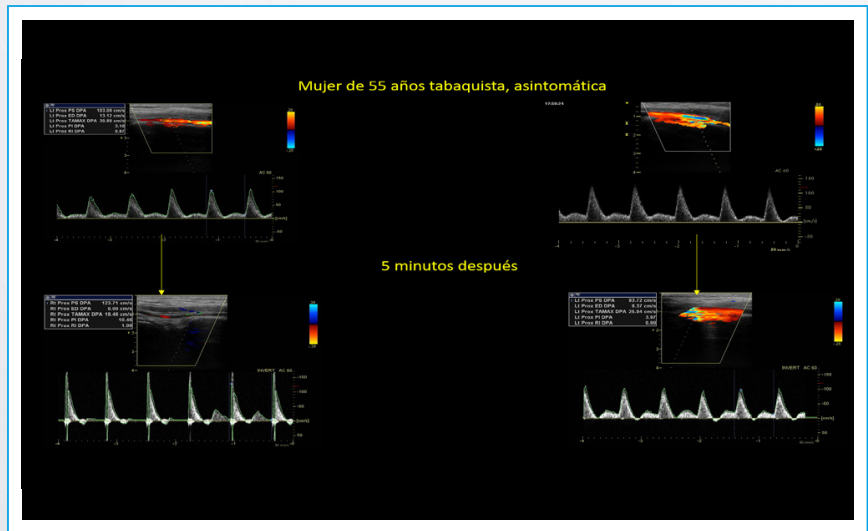

Figura 13. Misma paciente de la Figura 12. Pasados unos minutos, el flujo en las pedias es normal. Este fenómeno se da por vasodilatación, que reduce la resistencia periférica y el flujo distal pasa a ser monofásico 


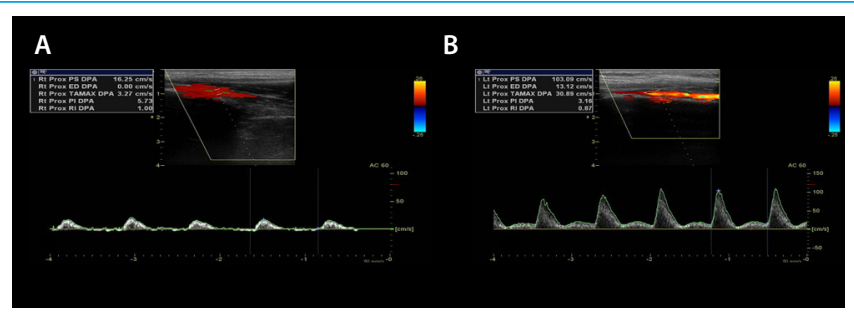

Figura 14. Diferencias entre un flujo monofásico de baja velocidad amortiguado por obstrucción previa (A) y un flujo monofásico por vasodilatación distal (B)

Por último, en la Figura 15 se observa una formación redondeada, con flujo en su interior, conectada a través de un estrecho cuello a la arteria femoral común (en este caso). El flujo en su interior es circular, formando un característico "yinyang", y a nivel de la comunicación entre ambas estructuras, donde se observa flujo turbulento, con aliasing, si uno coloca el Doppler pulsado, se observa la imangen típica de que todo el flujo que entra, sale (iguales volúmenes de flujo anterógrado y retrógrado). Es la imagen típica de los pseudoaneurismas.

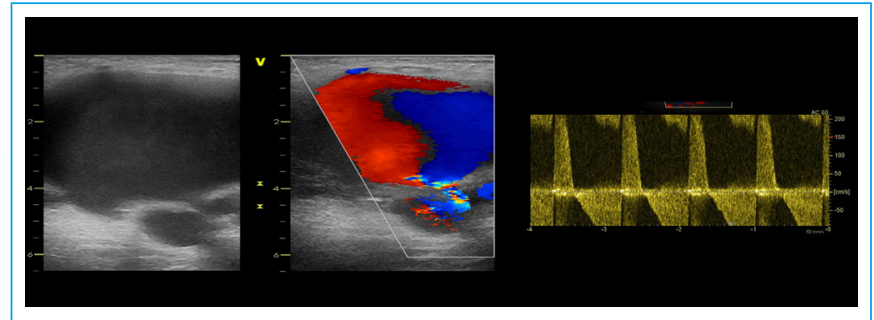

Figura 15. Imágenes ("yin-yang") y flujo sisto-diastólico típico de un pseudoaneurisma. Los pseudoaneurismas pueden formarse en cualquier lecho arterial, se caracterizan porque ha habido una ruptura incompleta de la pared y el hematoma es contenido por la adventicia o por el tejido circundante. En su interior el flujo es lento, se hallan coágulos, y pueden producirse como resultado de punciones (cateterismos, accesos a trayectos fistulosos) o traumatismos

\section{Conclusión}

Se muestran imágenes y flujos Doppler que deben conocerse, ya que guían hacia el diagnóstico, ayudando a decidir si existe patología subyacente o no, y direccionan soluciones terapéuticas. Se mostraron los flujos típicos normales y patológicos de las arterias de los vasos del cuello y miembros superiores e inferiores.

\section{Ideas para recordar}

- La ecografía Doppler vascular debe considerarse parte de la valoración cardiovascular integral.

- Es un método de bajo costo, reproducible, disponible y que puede realizarse con facilidad al lado del paciente.

- Es mandatorio conocer la morfología del flujo vascular en cada uno de sus territorios para intuir anormalidades en el momento en que se presentan.

\section{Bibliografía}

1. Flujo sanguíneo y su aspecto en la imagen de flujo en color. En: Thrush A y Harstshorne T. Ecografía Vascular. ¿Como, porqué y cuándo?. ELSEVIER Churchil Livingstone. Era. Ed. 2011. Cap. 5:49-63.
2. Arterias del Cuello. En Doppler de Cuello y Extremidades. Polak J. Marbán, Ed. 2007; Cap. 4:110-167

3. Forcada P. Aspectos biomecánicos de la circulación de la sangre. En: Esper R, Kotliar C, Bornotini M, Forcada P. Tratado de Mecánica Vascular e Hipertensión Arterial. Universidad Austral/Univesidad de Navarra. Ed. Intermédica-Buenos Aires 2010. Cap. 12:109-113.

4. Zwiebel W, Pellerito J. Zwiebel's Doppler General. Marbán, 5ta. Ed 2008, Cap.7: Hallazgos normales y aspectos técnicos de la ecografía carotídea:129- 139.

5. Arterias del Cuello. En Doppler de Cuello y Extremidades. Polak J. Cap. 4; Marbán, Ed. 2007; p110-167.

6. Taylor, Burns \& Wells. Aplicaciones Clínicas de la Ecografía Vascular. 2da. Ed. Marbán 2004.

7. Celermajer D, Sorensen K, Gooch V, et al. Non-invasive detection of endothelial dysfunction in children and adults at risk of atherosclerosis. Lancet 1992; 340:1111.

8. Zwiebel W, Pellerito J. Zwiebel's Conceptos básicos del análisis del espectro de frecuencias del Doppler y obtención de imágenes de flujo sanguíneo con ultrasonidos. En: Zwiebel's Doppler General. Marbán, 5ta. Ed 2008, Cap.3:59-85.

9. Spencer M, Reid J. Quantitation of Carotid Stenosis with ContinuousWave (C-W) Doppler Ultrasound. Stroke 1979;10(3): 326-330.

10. Aboyans V, Ricco J, Marie-Louise E, Bartelink M, Björck M, Brodmann M, et al. 2017 ESC Guidelines on the Diagnosis and Treatment of Peripheral Arterial Diseases, in collaboration with the European Society for Vascular Surgery (ESVS). Document covering atherosclerotic disease of extracranial carotid and vertebral, mesenteric, renal, upper and lower extremity arteries. Endorsed by: the European Stroke Organization (ESO). The Task Force for the Diagnosis and Treatment of Peripheral Arterial Diseases of the European Society of Cardiology (ESC) and of the European Society for Vascular Surgery (ESVS). Eur Heart J. 2018; 39:763-816.

11. Aboyans V, Ricco J, Marie-Louise E. Bartelink M, Björck M, Brodmann M, et al. 2017 ESC Guidelines on the Diagnosis and Treatment of Peripheral Arterial Diseases, in collaboration with the European Society for Vascular Surgery (ESVS). WebAddenda. Document covering atherosclerotic disease of extracranial carotid and vertebral, mesenteric, renal, upper and lower extremity arteries. Endorsed by: the European Stroke Organization (ESO). The Task Force for the Diagnosis and Treatment of Peripheral Arterial Diseases of the European Society of Cardiology (ESC) and of the European Society for Vascular Surgery (ESVS). Eur Heart J. 2017; 00:1-22.

12. Gerhard-Herman M, Gornik, H, Barrett C, et al. 2016 AHA/ACC Guideline on the Management of Patients With Lower Extremity Peripheral Artery Disease: Executive Summary A Report of the American College of Cardiology/American Heart AssociationTask Force on Clinical Practice Guidelines. Developed in Collaboration With the American Association of Cardiovascular and Pulmonary Rehabilitation, Inter-Society Consensus for the Management of Peripheral Arterial Disease, Society for Cardiovascular Angiography and Interventions, Society for Clinical Vascular Surgery,Society of Interventional Radiology, Society for Vascular Medicine, Society for Vascular Nursing, Society for Vascular Surgery, and Vascular and Endovascular Surgery Society. J Am Coll Cardiol 2017; 69:1465-508.

13. Gerhard-Herman M, Gornik, H, Barrett C, et al. 2016 AHA/ACC Guideline on the Management of Patients With Lower Extremity Peripheral Artery Disease. A Report of the American College of Cardiology/American Heart AssociationTask Force on Clinical Practice Guidelines. Developed in Collaboration With the American Association of Cardiovascular and Pulmonary Rehabilitation, InterSociety Consensus for the Management of Peripheral Arterial Disease, Society for Cardiovascular Angiography and Interventions, Society for Clinical Vascular Surgery, Society of Interventional Radiology, Society for Vascular Medicine, Society for Vascular 
Nursing, Society for Vascular Surgery, and Vascular and Endovascular Surgery Society. J Am Coll Cardiol 2017;69:e71-126.

14. Mozaffarian D, Benjamin E, Go A, Arnett D, et al. American Heart Association Statistics Committee and StrokeStatistics. Heart disease and stroke statistics- 2015 update: a report from the American Heart Association. Circulation 2015;131:e29-322.

15. Townsend N, Nichols M, Scarborough P, Rayner M. Cardiovascular disease in Europe 2015: epidemiological update. Eur Heart J 2015;36:2696.

16. Fernández-Friera L, Peñalvo J, Fernández-Ortiz A, Ibañez B, López-Melgar B, Laclaustra M, et al. Prevalence, Vascular Distribution, and Multiterritorial Extent of Subclinical Atherosclerosis in a Middle-Aged Cohort. The PESA (Progression of Early Subclinical Atherosclerosis) Study. Circulation 2015;131:2104-2113.

17. Parrot JD. The subclavian steal syndrome. Arch Surg 1969;88:661-5.

18. Kliewer M, Hertzberg B, Kim D, et al. Vertebral artery Doppler waveform changes indicating subclavian steal physiology. Am J Roentgenol 2000; 174:815- 9.

19. Ciancaglini C, D’Ovidio A. Protocolo para el estudio de la carótida interna extracraneal con eco Doppler Color. Rev Fed Arg Cardiol. 2013; 42(1): 65-70

20. Hiatt W, Brass E. Fisiopatología de la enfermedad arterial periférica, claudicación intermitente e isquemia crítica de la enfermedad. En: Medicina Vascular. Complemento de Braunwald. Tratado de Cardiología. ELSEVIER SAUNDERS 2da. Edición 2014;Cap.17:223-230.
21. CriquiMH,McClellandRL,McDermottMM,Allison MA, BlumenthalRS,Aboyans V, Ix JH, Burke GL, Liu K, Shea S. The ankle-brachial index and incident cardiovascular events in the MESA (Multi-Ethnic Study of Atherosclerosis). J Am Coll Cardiol 2010;56:1506-1512.

22. Vlachopoulos C, Xaplanteris P, Aboyans V, Brodmann M, Cifkova R, Cosentino $F$, et al. The role of vascular biomarkers for primary and secondary prevention. A position paper from the European Society of Cardiology Working Group on peripheral circulation: Endorsed by the Association for Research into Arterial Structure and Physiology (ARTERY) Society. Atherosclerosis 2015;241:507-532.

23. Peripheral Arteries. En: Schäberle W. Ultrasonography in Vascular Diagnosis. A Therapy-Oriented Textbook and Atlas. Springer-Verlag Berlin Heidelberg 2005; Ch. 2:29-110

24. Thrush A \& Hartshone T. Peripheral Vascular Ultrasound. Elsevier Churchil Livingstone2006.

25. Mohler III ER, Gornik HL, Gerhard-Herman M, et al. ACCF/ACR/AIUM/ASE/ ASN/ICAVL/SCAI/SCCT/SIR/SVM/SVS 2012 Appropriate Use Criteria for Peripheral Vascular Ultrasound and Physiological Testing Part I:Arterial UItrasound and Physiological Testing. J Am Coll Cardiol. 2012,60(3):242- 276

26. Creager A, Libby P. Peripheral Artery Diseases. Braunwald's Heart Diseases. Elsevier Saunders. 10th. Edition 2015; Ch 58:1312-1335. 27. Henericci M. Diagnóstico Vascular con Ultrasonido. Amolca. 2da. Ed. 2009.

27. Henericci M. Diagnóstico vascular con Ultrasonido. Amolca. 2da. Ed. 2009 